\title{
Supervisory Fault Tolerant Control of the NASA AirStar Aircraft*
}

\author{
Tamás Péni ${ }^{1,2}$, Bálint Vanek ${ }^{1}$, Zoltán Szabó ${ }^{1}$ and József Bokor ${ }^{1}$
}

\begin{abstract}
A multi-level reconfiguration framework is proposed for fault tolerant control of overactuated aerial vehicles, where the levels indicate how much authority is given to the reconfiguration task. On the lowest, first level the fault is accommodated by modifying only the actuator/sensor configuration, so the fault remains hidden from the baseline controller. A dynamic reallocation scheme is applied on this level. The allocation mechanism exploits the actuator/sensor redundancy available on the aircraft. In case the fault cannot be managed at the actuator/sensor level the reconfiguration process has access the baseline controller. Based on the LPV control framework, this is done by introducing fault-specific scheduling parameters. The baseline controller is designed to provide acceptable performance level along all fault scenarios coded in these scheduling variables. The decision of which reconfiguration level has to be initiated in response to a fault is determined by a supervisor unit. The method is demonstrated on the full six degrees of freedom nonlinear simulation model of the GTM UAV.
\end{abstract}

\section{INTRODUCTION}

A major goal in modern flight control system research is the need to pursue improved reliability and environmental sustainability of safety critical systems [7]. Faults and failures may result in loss of performance and even in catastrophic harm or loss of life. Reconfigurable control methods promise a way to compensate for failures or damage of flight control effectors by using the remaining flight control surfaces to generate compensating forces and moments, via changes in the flight control algorithms. The majority of faults can be detected immediately with system checks, but a few of them requires fault detection and isolation methods (FDI). Combined with reconfigurable control methods they are considered as active fault tolerant control (FTC), which assumes a well defined mechanism to actively detect and counteract against faults in the system [12], [9]. In passive FTC controllers are fixed and are designed to be robust against a class of presumed faults. This approach does not need a fault estimate (or detection) or controller reconfiguration, but provides only limited fault-tolerant capabilities [14].

In general, a fault tolerant flight control system is required to perform failure detection, identification and accommodation for sensor and actuator failures. Active FTC schemes can be broadly classified into projection based and online controller redesign based approaches [11]. Numerous results have been proposed related to both approaches in the past few years [5], [1]. More recently in a numerous research projects, within the EU FP7 framework, focus on the environmental impact of the aircraft and hence higher performance is required from the flight control system, with certifiable algorithms, leading to a paradigm shift from robust passive FTC towards active methods relying on switching, gain scheduled or linear parameter-varying (LPV) methods.

The aim of the paper is to present a multi level method combining dynamic control allocation and control reconfiguration. The baseline control system is designed for the nominal dynamics of the aircraft, while faults and actuator saturation limits are handled by the dynamic control allocation scheme in case they pose a feasible challenge given the original handling-qualities. In case the level of system degradation is beyond the limits of the original control system the handling-qualities have to be revised and

\footnotetext{
*The research leading to these results has received funding from the European Union's Seventh Framework Programme (FP7/2007-2013) under grant agreement no. FP7 - AAT - 2012 - 314544

1 The authors are with MTA SZTAKI, Institute for Computer Science and Control, Hungarian Academy of Sciences, Budapest, Hungary

${ }^{2}$ corresponding author, email: pt@scl.sztaki.hu
}

lower performance objectives along with increased robustness have to be provided for the faulty system. Coordination of these components is provided by a supervisor which re-allocates control authority based on health information, flight envelope limits and cross coupling between lateral and longitudinal motion. The monitoring components and FDI filters provide the supervisor with information about different fault operations, based on that it is able to make decisions about necessary interventions into the vehicle motions and guarantee fault-tolerant operation of the aircraft. The design of the proposed reconfigurable control algorithm is based on Linear Parameter-varying (LPV) control methods that uses a parameter dependent dynamic control allocation scheme. The prime advantage of this approach is that the synthesis results in a single multivariable controller with stability and robustness guarantees for the closed-loop system. The design is demonstrated on the full six degrees-of-freedom highfidelity simulator of the NASA AirSTAR Flight Test Vehicle. The paper is organized as follows. Section II presents a brief introduction to the supervisory architecture. The vehicle model of the NASA AIRStar UAV are presented in Section III together with the baseline controller synthesis. The dynamic input allocator is described in Section IV, which is followed by simulation results in Section V. Conclusions are drawn in Section VI.

\section{TWO-LEVEL RECONFIGURABLE CONTROL}

The reconfiguration framework proposed in the paper is based on a multi-level approach. The subsequent levels indicate how much authority is given to the reconfiguration task. On the lowest, first level, the effect of the fault is not significant and moderate maneuvers are commanded, hence the fault is accommodated by modifying only the actuator/sensor configuration. In this case the fault remains hidden from the baseline controller. This is an important requirement in commercial aviation [6] from the perspective of the pilot and from the Flight Control System (FCC), since only the flight envelope constraints might be impacted, but the autopilot functions can remain unchanged. A dynamic reallocation scheme, e.g. the one proposed in [13], can be extended and applied on this level as proposed in Section IV. The allocation mechanism exploits the actuator/sensor redundancy available on the aircraft. It has already been showed e.g. in [8] that most critical actuator/sensor faults can be managed in this way, therefore we expect that most of the possible failures will be successfully accommodated without modifying the baseline controller.

If a fault cannot be managed at the actuator/sensor level then the reconfiguration process has to be allowed to access the baseline controller. This can be achieved via the fault specific scheduling parameter $\rho_{f}$, detailed in Section III-C.

The decision on how the control system reconfigures in response to a faults is determined by a supervisor unit. Methods using controlled invariant set computation [2] can be employed to systematically study the region of stability in various fault cases. In the present method a simple logic is used. In case of a single jamming fault of a surface, only Level-1 is used, while faults affecting multiple surfaces trigger Level-2 $\left(\rho_{f}=1\right)$ besides Level-1. The supervisor is also responsible for generating and providing all necessary fault information (surface affected, type of fault, failed position, etc.) to the reconfiguration subsystems: the supervisor manages the actuator/sensor reallocation procedure and constructs the fault-dependent scheduling parameters for the scheduled baseline controller. The entire control architecture is depicted in Figure 1. 


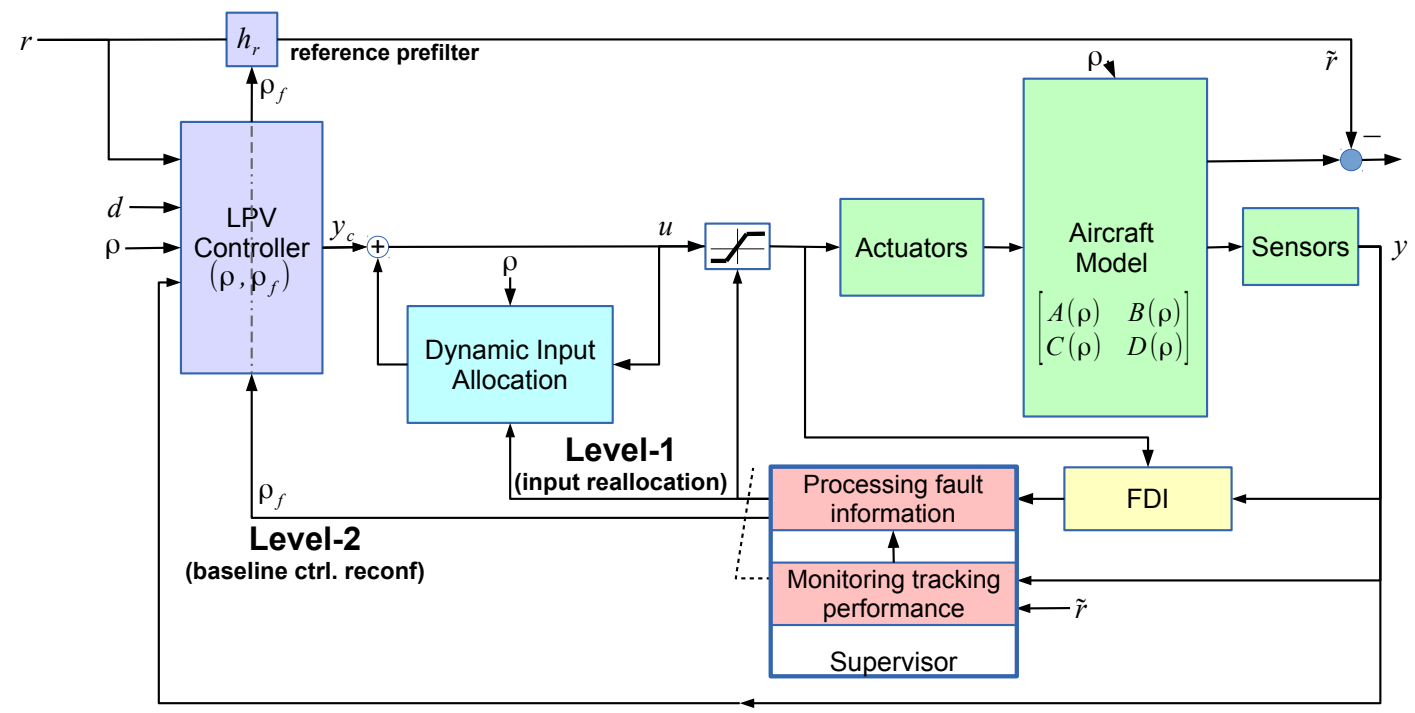

Fig. 1. General architecture of multi-level reconfigurable control

\section{AIRCRAFT MODEL AND BASELINE
CONTROL DESIGN}

An effective resource for experimentally testing fight control algorithms, including adaptive control algorithms, is the Airborne Subscale Transport Aircraft Research (AirSTAR) testbed at NASA Langley Research Center [10]. The primary AirSTAR flight test vehicle is a turbine powered $5.5 \%$ dynamically scaled model of a civilian transport aircraft, often referred to as the Generic Transport Model (GTM). The GTM has a wing span of $7 \mathrm{ft}$, and weighs around $55 \mathrm{lbs}$. Under normal operations, it flies at an altitude of 700 to $1100 \mathrm{ft}$, with an airspeed between 70 and 85 knots. The currently used T-2 test aircraft is shown in Figure 2. Significant wind tunnel and flight testing has been performed to identify the flight dynamics of the GTM [3]. A nonlinear simulation model of the aircraft dynamics has been developed and is readily available to the research community. Experimental control algorithms are easily embedded in this simulation model for verification prior to flight testing [4]. Hence, the AirSTAR testbed is a highly effective for experimental flight control research through its flexible architecture and rapid implementation and testing cycle. To fully understand the

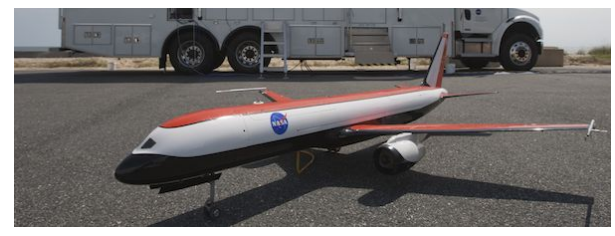

Fig. 2. The NASA AIRStar vehicle.

theoretical and practical aspects of the fault tolerant control scheme the rigid body control of the aircraft motion has to be designed for both lateral and longitudinal planes. After obtaining a suitable LPV model, a baseline LPV control system is designed for pitch angle $\theta$ and airspeed $V_{\text {cas }}$ control in the longitudinal, and an advanced LPV controller for roll angle $\phi$ and sideslip angle $\beta$ control in the lateral plane.

\section{A. Lateral model of GTM aircraft}

A four state model, decoupled from the eight state reduced-order model, captures the primary lateral flight dynamics of the GTM. The states of the model are sideslip angle $\beta[\mathrm{rad}]$, roll rate $p[\mathrm{rad} / \mathrm{s}]$, yaw rate $r[\mathrm{rad} / \mathrm{s}]$, and roll angle $\phi[\mathrm{rad}]$. The control inputs to the model have significant redundancy, left and right aileron deflection $\delta_{a, L}, \delta_{a, R}[\mathrm{rad}]$, upper and lower rudder $\delta_{r, U}, \delta_{r, L}[\mathrm{rad}]$, inner and outer spoiler deflection $\delta_{s p, I}, \delta_{s p, O}[\mathrm{rad}]$, and left, right throttle $\delta_{T H R, L}, \delta_{T H R, R}[\%]$. Due to the physical limits of the spoilers and their symmetric effect on the lateral dynamics the control inputs are sent to the left spoilers in case of positive demand and to the right spoilers in case of opposite demand. The lateral LPV state-space model of the GTM between 60 and 100 knots is approximated with an affine LPV model in the form of:

$$
\left[\begin{array}{c}
\dot{\beta} \\
\dot{p} \\
\dot{r} \\
\dot{\phi}
\end{array}\right]=\left(A_{0}+A_{V} V_{\text {cas }}\right)\left[\begin{array}{c}
\beta \\
p \\
r \\
\psi
\end{array}\right]+\left(B_{0}+B_{V} V_{\text {cas }}\right)\left[\begin{array}{c}
\delta_{a, L} \\
\delta_{a, R} \\
\delta_{r, U} \\
\delta_{r, L} \\
\delta_{s p, I} \\
\delta_{s p, O} \\
\delta_{T H R, L} \\
\delta_{T H R, R}
\end{array}\right]
$$

with outputs of all four states, where the $C=\frac{180}{\pi} I_{4 \times 4}$ matrix is constant. The $A_{0}, A_{V}, B_{0}, B_{V}$ affine LPV coefficients are obtained with least square fit on the pointwise LTI plants. The plant is augmented with first order actuator dynamics of $G_{a c t}=\frac{10 \pi}{s+10 \pi}$ on ailerons, rudders and spoilers and $G_{e n g}=$ $\frac{-0.1474 s+0.7314}{s^{2}+1.336 s+0.7314}$ on the throttle, which contains a right half plane zero. Sensor dynamics are omitted due to the high quality of the sensors onboard. An additional input is the right aileron fault, which has the same input direction as the aileron input, but the fault signal is the opposite of the aileron command, leading to the cancellation of the second column in the corresponding $B$ matrix in case of jamming.

\section{B. Longitudinal model of GTM aircraft}

The remaining four states, decoupled from the eight state reduced-order model, captures the primary longitudinal flight dynamics of the GTM. The states of the model are pitch angle $\theta[\mathrm{rad}]$, pitch rate $q[\mathrm{rad} / \mathrm{s}]$, angle of attack $\alpha[\mathrm{rad}]$, and calibrated airspeed $V_{c a s}[\mathrm{~m} / \mathrm{s}]$. The control inputs to the model have significant redundancy also, left and right elevator deflection $\delta_{e, L}, \delta_{e, R}[\mathrm{rad}]$, inner and outer spoiler deflection on both sides $\delta_{s p, I}, \delta_{s p, O}[\mathrm{rad}]$, and left, right throttle $\delta_{T H R, L}, \delta_{T H R, R}[\%]$. Due to the physical limits of the spoilers and their effect on the longitudinal dynamics the control inputs are sign constrained, which have to be taken care by the control allocation method. The longitudinal LPV state-space model of the GTM between 60 and 100 knots is approximated with an affine LPV model in the form similar 
to the lateral dynamics (Eq. 1), hence the details are omitted here. Measured outputs are the four states, where the $C$ matrix is constant. The $A_{0, \text { lon }}, A_{V \text {,lon }}, B_{0, l o n}, B_{V, \text { lon }}$ affine LPV coefficients are obtained with least square fit on the pointwise LTI plants. The plant is augmented with the same actuator dynamics on elevators and spoilers as on the lateral control inputs, and the same engine dynamics described above is also used on the throttle. No fault is assumed on the longitudinal dynamics for the present investigation to keep the overall system complexity tractable. Sensor dynamics on all eight measured outputs are omitted due to the high quality of the sensors onboard, but due to the onboard communication and digital implementation of the controllers a time delay of $0.03 \mathrm{~s}$ is assumed on all sensor channels, which is accounted by $4^{\text {th }}$ order Padé approximation.

\section{Baseline Controller Design with fault-dependent schedul- ing}

The system interconnection (Fig. 3) addressing the parameter dependent controller synthesis proposed to solve the roll angle and sideslip angle tracking problem, assuming noise and exogenous disturbances, is detailed in the following. The goal of the controller synthesis is to have robust performance across all operating points. To account for system health information the LPV representation, scheduled with $\rho_{V}=V_{c a s}$ is augmented with a fault specific scheduling variable $\rho_{f}=[0 ; 1]$. This allows to trade off high performance in healthy operation $\left(\rho_{f}=0\right)$ with robust, less demanding behavior in case of failures $\left(\rho_{f} \leq 1\right)$. Model matching is achieved by filtering the reference signal through a "handling-qualities" model, scheduled with $\rho_{f}$, to achieve smooth behavior with adequate speed of response for bank angle and side speed commands. The main control objectives, to keep the error between the plant outputs $\phi, \beta$ and the desired handling-quality responses $h q_{\phi}=\left(\frac{2.5-\rho_{f}}{s+2.5-\rho_{f}}\right)^{2} ; h q_{\beta}=\left(\frac{1.5-\rho_{f}}{s+1.5-\rho_{f}}\right)^{2}$ low are weighted across frequency and health status with $W_{\phi}=$ $\left(7-2 \rho_{f}\right)\left(\frac{3.5-2 \rho_{f}}{s+3.5-2 \rho_{f}}\right)^{2} ; W_{\beta}=\left(3-2 \rho_{f}\right)\left(\frac{1.5-0.75 \rho_{f}}{s+1.5-0.75 \rho_{f}}\right)^{2}$ across all parameter range, trading off good steady state tracking with degraded performance at frequencies higher than $5 \mathrm{rad} / \mathrm{s}$. The speed of response and tracking error requirement is reduced at higher frequencies as the aircraft is subjected to faults, which has direct impact on the control authority requirement in transient modes, especially when the reference command is changing. Actuator usage is penalized in the design with weights of $W_{a c t}=(3+$ $\left.2 \rho_{f}\right) \operatorname{diag}(1 / 20 ; 1 / 20 ; 1 / 30 ; 1 / 30 ; 1 / 15 ; 1 / 45 ; 1 / 25 ; 1 / 25)$, corresponding to the maximum actuator deflections respecting the physical limits of the individual control effectors, while also addressing the increased actuator usage during faults. Characteristics of the noise is captured by a weight of $W_{n}=\left(3+2 \rho_{f}\right) \operatorname{diag}(0.04 ; 0.1 ; 0.1 ; 0.04)$ with fault dependent magnitude, accounting for higher uncertainty. But the weight is constant across frequency, and assumes higher noise on pitch and yaw rate sensors than on bank angle due to the sensor characteristics.

The weights are optimized with linear point design first, at 18 points of the parameter space $\left[\rho_{V} ; \rho_{f}\right]=[60: 5: 100 ; 0$ : $1]$ in the LPV model. During the pointwise $\mathcal{H}_{\infty}$ synthesis the $\gamma$ performance level ranges between 0.835 and 1.001 , with lower values at lower speeds and at $\rho_{f}=1$, where lower performance is required. The LPV synthesis with unbounded parameter rate, with constant Lyapunov function leads to a higher $\gamma$ performance level, the $\mathcal{L}_{2}$ gain is 1.1 . This is a consequence of using a single, parameter dependent LPV controller, where the parameter rates can be unbounded. The longitudinal LPV controller is designed in a similar way, but without fault scheduling for the tracking of and $\theta$. $V_{\text {cas }}$ commands are followed using a setpoint tracking scheme, since $V_{c}$ as is also used as scheduling variable, hence the trim velocity is always the measured one and the tracking error is always zero.
In the baseline control design all actuators are assumed to be used, with their maximum deflection limits, and the control allocation is assigning the correct amount respecting the supervisory commands, according to the health status and saturation of the actuators.

\section{DYNAMIC INPUT REALLOCATION}

This section extends the input reallocation method proposed in [13] to parameter varying plants driven by dynamical actuators. In [13] only LTI models are assumed and the controller acts directly on the plant. This section considers the configuration when LTI actuators are connected to an LPV plant and the reconfiguration has to be performed through the actuator dynamics. Let the actuator models be collected in a strictly proper, linear, time-varying state-space model as follows:

$$
\begin{aligned}
& \dot{x}_{a}=A_{a} x_{a}+B_{a} u_{a} \\
& y_{a}=C_{a} x_{a}
\end{aligned}
$$

Here $x_{a}$ collects the states of all actuators and $u_{a}, y_{a}$ denote the inputs and the outputs, respectively. Since the plant is driven by the actuators, the input to the plant $(u)$ equals $y_{a}$. In nominal case, $u_{a}$ equals the output of the controller, i.e. $u_{a}=$ $y_{c}$. Input reallocation is performed if some failure occurs and thus the control effort has to be redistributed among the healthy actuators. To modify the control input combination, an additional signal $\tilde{u}_{a}$ will be designed so that $y_{c}+\tilde{u}_{a}$ converges to the required configuration while $\tilde{u}_{a}$ remains totally unseen from the plant. (The latter requirement ensures that the reconfiguration does not affect the nominal closed loop performance.) These goals are intended to be achieved by exploiting the input redundancy of the system, which is available if $\operatorname{rank}\left(B_{u}(\rho)\right)<n_{u}, \forall \rho$.

We will assume that the baseline controller has been designed for the input-redundant augmented plant containing the actuators as well. (This approach differs from those methods [8], where controller is designed to generate only torques and forces and thus the actuator dynamics are not explicitly taken into consideration during the synthesis procedure.)

The first step towards allocator design is to characterize those inputs $u_{a}$, which does not have any effect on the plant. To this end, we start from the dynamics

$$
\dot{x}=A(\rho) x+B_{u}(\rho) y_{a}=A(\rho) x+B_{u}(\rho) C_{a} x_{a}
$$

Let $B(\rho)$ be defined so that its rows span the same (parameter-dependent) subspace than $B_{u}(\rho)$, but let $B(\rho)$ have full row-rank. Define $v(\rho)=B(\rho) C_{a} x_{a}$ as a new output for the actuator dynamics and let $r=\left(r_{1}, \ldots, r_{n_{v}}\right)$ be the vector relative degree of the modified system

$$
\begin{aligned}
\dot{x}_{a} & =A_{a} x_{a}+B_{a} u_{a} \\
v & =B(\rho) C_{a} x_{a}
\end{aligned}
$$

Then this model can be rewritten as follows:

$$
\begin{aligned}
z_{1}^{i, 1} & =v_{i} \\
\dot{z}_{1}^{i, 1} & =z_{1}^{i, 2} \\
\vdots & \\
\dot{z}_{1}^{i, r_{i}-1} & =z_{1}^{i, r_{i}} \\
\dot{z}_{1}^{i, r_{i}} & =a_{1, i}\left(\eta_{r_{i}}\right)^{T}\left[\begin{array}{c}
z_{1} \\
z_{2}
\end{array}\right]+b_{1, i}\left(\eta_{r_{i}}\right)^{T} u_{a}^{j} \\
\dot{z}_{2} & =A_{2}\left(\eta_{\max r_{i}}\right)\left[\begin{array}{c}
z_{1} \\
z_{2}
\end{array}\right]+B_{2}\left(\eta_{\max r_{i}}\right) u_{a}
\end{aligned}
$$

where $\eta_{s}=\left(\rho, \dot{\rho}, \ldots, \rho^{(s)}\right)$ and $z_{2}$ is chosen so that the mapping $T: x_{a} \rightarrow\left[\begin{array}{l}z_{1} \\ z_{2}\end{array}\right]$ is invertible. By setting

$$
u_{a}=w_{p}+\operatorname{ker}\left(B_{1}\right) w
$$

with $w_{p}$ satisfying $A_{1}\left[\begin{array}{l}z_{1} \\ z_{2}\end{array}\right]+B_{1} u_{a}=0$ then system (5) is transformed into a chain of integrators producing zero output for any input $w$.

In this paper we are going to follow this procedure to construct the reconfiguration signal, but in order to ease the discussion we make the following assumption: 


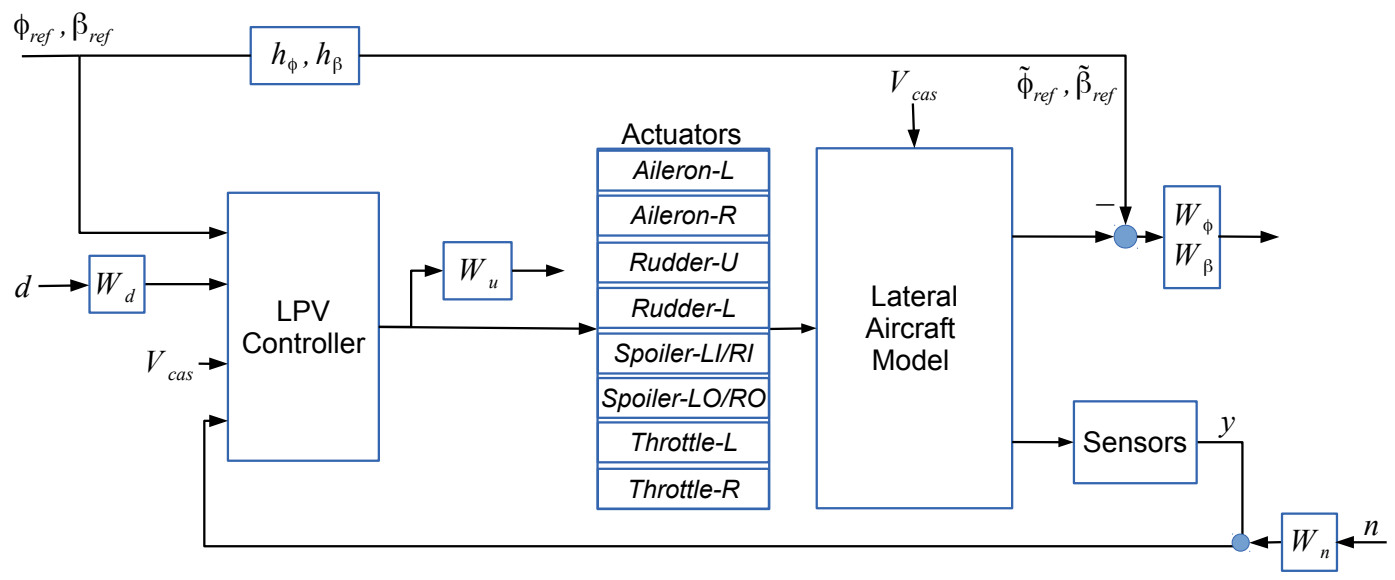

Fig. 3. Block diagram of baseline lateral control design for GTM aircraft

Assumption. 1: The vector relative degree of (3) is $(1,1,1, \ldots, 1)$, i.e. the relative degree from each output $v_{i}(\rho)$ is 1 .

In practice, this assumption is not too restrictive as most actuators can be well-approximated by a 1 or 2 dimensional models having explicit input dependence in the first derivatives of the output. For an example, see the case study in section V.

If Assumption 1 holds we can introduce the following coordinate transformation:

$$
T(\rho)=\left[\begin{array}{c}
B(\rho) C_{a} \\
T^{\prime}(\rho)
\end{array}\right], \quad z_{a}=T(\rho) x_{a}=\left[\begin{array}{c}
z_{1} \\
z_{2}
\end{array}\right], \quad z_{1}=v
$$

where $T^{\prime}(\rho)$ is chosen so that $T(\rho)$ is invertible for all $\rho$. Then we have

$$
\dot{z}_{a}=\left[\frac{\partial T(\rho)}{\partial \rho} \dot{\rho}+T(\rho) A_{a}\right] T(\rho)^{-1} z_{a}+T(\rho) B(\rho) u_{a}
$$

i.e.

$$
\begin{aligned}
& \dot{z}_{1}=A_{1}(\rho, \dot{\rho}) z_{a}+B_{1}(\rho) u_{a} \\
& \dot{z}_{2}=A_{2}(\rho, \dot{\rho}) z_{a}+B_{2}(\rho) u_{a}
\end{aligned}
$$

If

$$
\tilde{u}_{a}=w_{p}(\rho, \dot{\rho}, z)+\operatorname{ker}\left(B_{1}(\rho)\right) w
$$

where $w$ is arbitrary and $w_{p}$ satisfies

$$
A_{1}(\rho, \dot{\rho}) z_{a}+B_{1}(\rho) w_{p}=0
$$

then the dynamics (6a) at $u_{a}=\tilde{u}_{a}$ will simplify to $\dot{z}_{1}=0$, i.e. $z_{1}$ remains zero for all time. As a consequence, $(6 \mathrm{~b})$ will depend only on $z_{2}$, that is

$$
\begin{aligned}
\dot{z}_{2}= & A_{2}(\rho, \dot{\rho})\left[\begin{array}{c}
0 \\
z_{2}
\end{array}\right] \\
& +B_{2}(\rho)\left(w_{p}\left(\rho, \dot{\rho}, z_{2}\right)+\operatorname{ker}\left(B_{1}(\rho)\right) w\right)
\end{aligned}
$$

Since (2) is always stable, (8) (which is the zero-dynamics of (6)) is stable as well.

Summarizing the result, the modified control input $\tilde{u}_{a}$ is computed in the following way: (8) generates $z_{2}, z_{a}=\left[\begin{array}{c}0 \\ z_{2}\end{array}\right]$ and $w_{p}$ is determined from (7). Then

$$
u_{a}=y_{c}+w_{p}\left(\rho, \dot{\rho}, z_{2}\right)+\operatorname{ker}\left(B_{1}(\rho)\right) w
$$

It is important to emphasize that $z_{2}$ is generated by the allocator, independently from the actuator's state. Therefore, we do not need to measure $x_{a}$ in order to compute the reconfiguration signal. This is because $w_{p}$ is designed to compensate the effect of $w$ and not the effect of $y_{c}$. If the model of the actuator is known up to an acceptable precision then $z_{2}$ precisely approximates the effect of $w$ on the real actuators.

The remaining component of the compensator is the reconfiguration signal $w$, which is constructed similarly to the LTI case:

$$
\dot{w}=-K \operatorname{ker}\left(B_{1}(\rho)\right)^{T} W u_{a}
$$

Here $W$ is the (diagonal) weighting matrix controlling the contribution of each actuator and $K$ is a feedback gain rendering (9) stable. It can be proved [13] that at constant parameter-control-output pair $\left(\rho^{*}, y_{c}^{*}\right) w$ converges to the optimal value minimizing the quadratic cost function

$$
J(w)=\left(y_{c}^{*}+\operatorname{ker} B_{1}\left(\rho^{*}\right) w\right)^{T} W\left(y_{c}^{*}+\operatorname{ker} B_{1}\left(\rho^{*}\right) w\right)
$$

As for the entries of $W$, a large value at $W_{i, i}$ means switching off the $i$-th actuator. If the $i$-th actuator have upper and lower limits (e.g. $-M_{i}$ and $M_{i}$ ) which cannot be exceeded then $W_{i i}$ can be chosen as follows [13]:

$$
W_{i i}=\left[(1+\epsilon) M_{i}-\left|\operatorname{sat}_{M_{i}}\left(u_{a}(i)\right)\right|\right]^{-1}
$$

\section{SIMULATION RESULTS}

In order to illustrate the relevance of the two-level reconfiguration architecture, a rudder fault scenario is investigated on the GTM, where both levels of controller reconfiguration have to be activated to accommodate the fault.

First we analyze the performance of the control allocation method on a single (upper) rudder jamming case. To perform the reconfiguration tasks we assume that the fault has been detected and precise fault information is available for the supervisor unit. In response to the fault, the supervisor immediately switches off the rudder (at 0 position) and reconfigures the controller to attenuate the effect of actuator loss (see Fig. 4).

The control task is a simultaneous doublet tracking problem on both $\beta$ and $\phi$ as shown in Figure 5, where minor tracking error can be observed due to the combined maneuver, where cross-coupling is fundamentally difficult to handle. Notice that the aircraft has calibrated airspeed of 80 knots at the beginning of the maneuver in each simulation case, which slightly decreases towards the end since velocity control has slower response. It can be stated that the input allocator efficiently handles the loss of the surface, tracking of both reference signals are good. On the other hand, the baseline controller alone would provide adequate $\phi$ tracking with unacceptably poor $\beta$ response.

In what follows we analyze the case, when both rudders fail and have to be switched off. To compare the results of the different control configurations, the first simulation is performed without any reconfiguration. In this case the baseline controller tries to control the faulty aircraft. It can be seen in Fig. 6 and Fig. 7 that the capability of tracking $\beta$-reference is lost.

Fig. 8 and Fig. 9 presents the flight results obtained by using only local control allocation. To compensate the faulty rudders the allocator tries to generate larger inputs on the healthy actuators, but this is prevented by the limit 


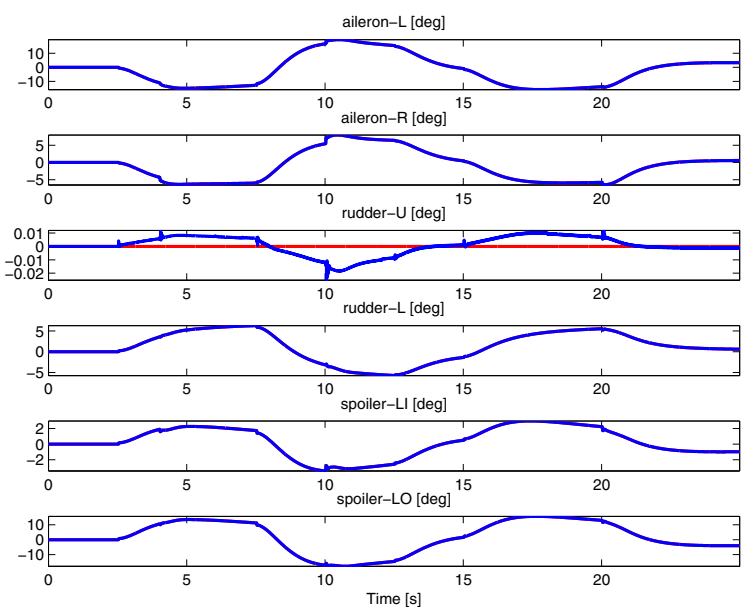

Fig. 4. First 6 control inputs in upper rudder jamming fault case, input allocation with baseline controller. Blue: the output of the controller. Red: the saturated input signals.
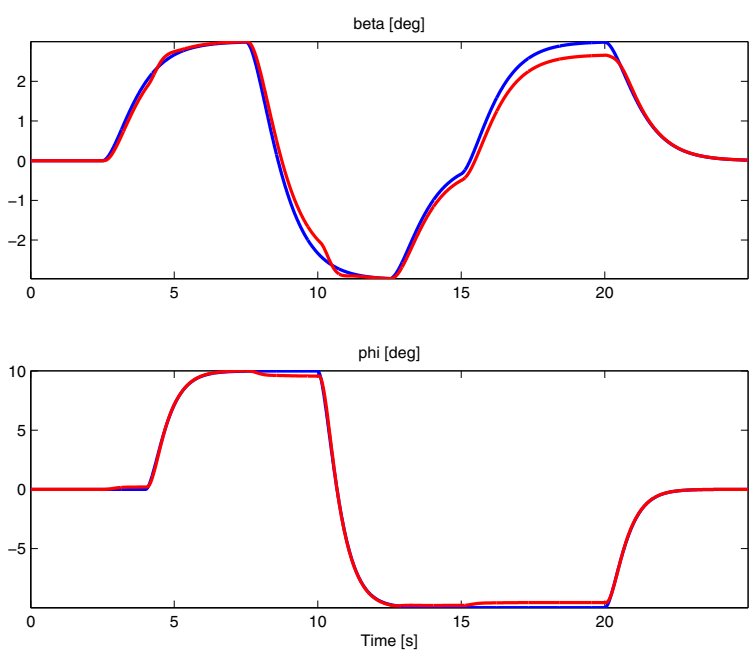

Fig. 5. Output tracking in upper rudder jamming fault case, input allocation with baseline controller. Blue: reference signals filtered by $h_{\beta}, h_{\phi}$. Red: outputs of the GTM model.

constraints (10). These two conflicting goals generate a tradeoff in the allocator dynamics, which leads to a significant performance degradation and loss of stability. (During the simulation runs, the actuator limits introduced in section III-C are applied, i.e. $M=[20,20,-,-, 15,45,25,25]$ are used in (10). The third and fourth diagonal entries of $W$, which correspond to the inactive rudders are set to 1000 . This large weight is used to decrease the contribution of the faulty components of the control input vector. The remaining parameters of the allocator are chosen as follows: $\epsilon=0.01$, $K=\operatorname{diag}(0.1,0.1, \ldots, 0.1)$.

Simulation are also performed to see how setting $\rho_{f}$ scheduling variable of the LPV controller to 1 affects the response alone. By shifting $\rho_{f}$ from 0 (nominal case) to 1 (faulty case) the required speed of convergence is adjusted. At $\rho_{f}=0$ fast handling quality filters are set, which means fast systems response in nominal case. As $\rho_{f}$ is shifted towards 1 the poles of the prefilters decrease, which means slower reference tracking for the faulty aircraft. The results show that the double rudder fault cannot be accommodated by the controller scheduling alone, the ability to track $\beta$ reference is still lost.

Finally, Figs. 10 and 11 present the simulation results,
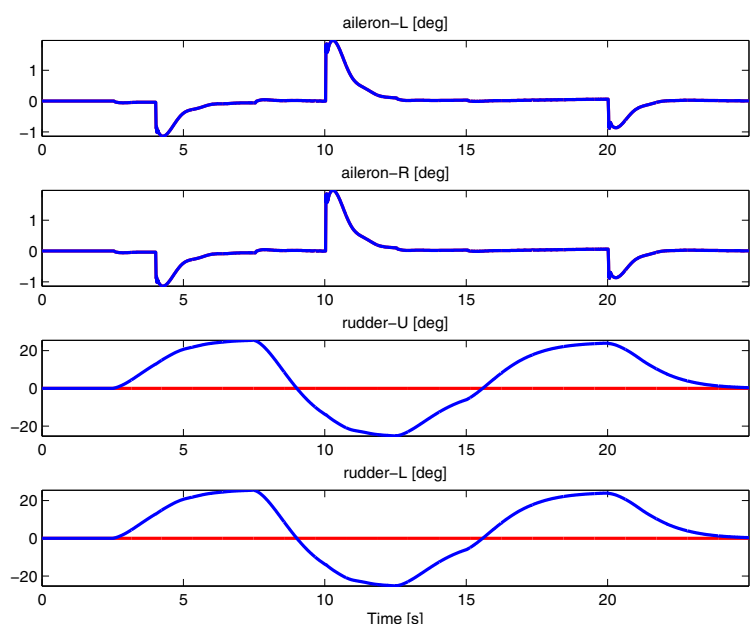

Fig. 6. First 4 control inputs in complete rudder jamming case with baseline controller. Blue: the output of the controller. Red: the saturated input signals (The rudders are switched off.)
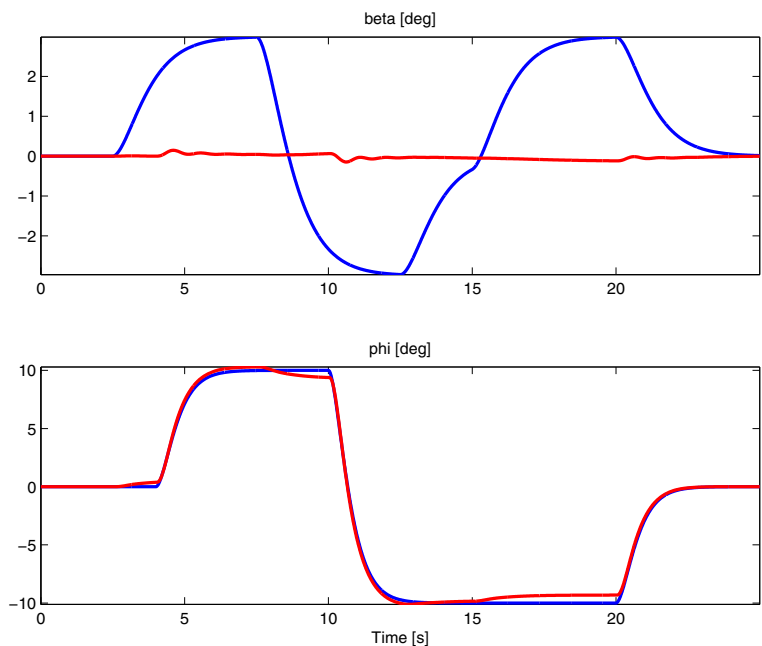

Fig. 7. Output tracking in complete rudder jamming case with baseline controller. Blue: reference signals filtered by $h_{\beta}, h_{\phi}$. Red: outputs of the GTM model.

when both the input reallocation and the fault-dependent scheduling are applied. Compared to the previous simulation scenarios, the improvement is significant. The aircraft managed to track both reference signals with acceptably small tracking errors. Errors can be observed due to the demanding nature of the problem and left aileron commands are reaching the saturation limits for longer periods, but the plane remains controllable in both $\phi$ and $\beta$ axes.

\section{CONCLUSIONS}

The present paper shows the advantages of the proposed two-level reconfigurable control approach based on control allocation and LPV performance scheduling. Due to the hardware redundancy of actuation surfaces onboard the current aircraft it is often advantageous to re-allocate the control authority among the healthy actuators, but mostly it is done in a discrete way with pre-programmed routines which have to be tailored to each individual fault case, while the dynamic input allocation method presented here is fairly general to handle various kinds of failures within a common framework. On the other hand, in certain fault cases, the original handling qualities requirements are no longer feasible do to reduced 

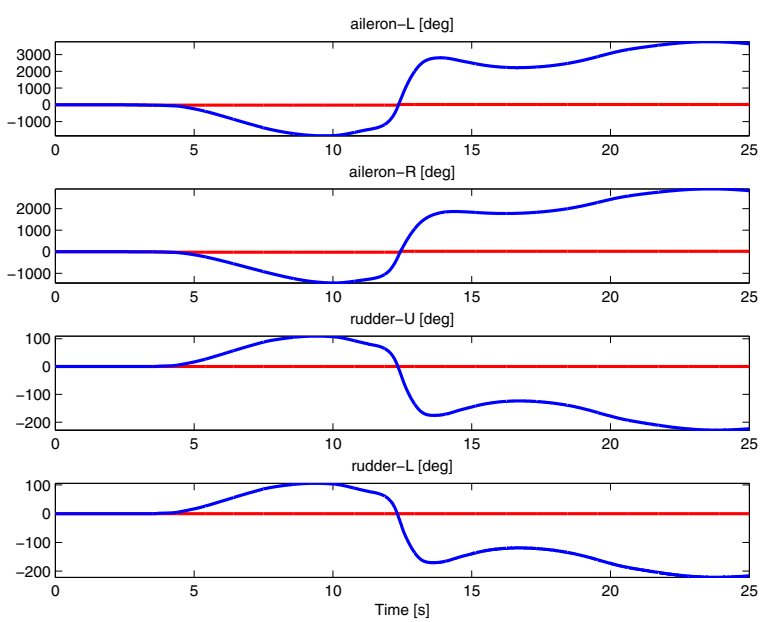

Fig. 8. First 4 control inputs in complete rudder jamming case using only input allocation. Blue: modified control inputs. Red: the saturated input signals. (The rudders are switched off.)
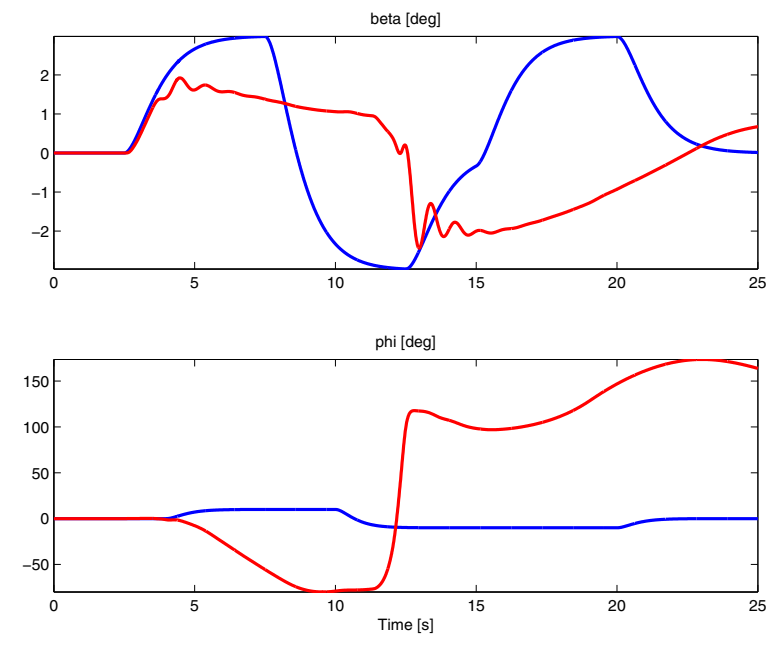

Fig. 9. Output tracking in complete rudder jamming case using only input allocation. Blue: reference signals filtered by $h_{\beta}, h_{\phi}$. Red: outputs of the GTM model.

authority, hence a graceful degradation of the performance is inevitable, which is scheduled with an LPV controller tuned to trade-off performance vs. robustness. The complimentary properties of the two reconfiguration methods are highlighted in the paper with simulation results, where lateral commands are followed with various rudder fault conditions.

\section{ACKNOWLEDGMENT}

The authors greatly acknowledge the help of Dr. Irene Gregory and David Cox of NASA Langley Research Center for making the GTM simulation model available for the purpose of this research.

\section{REFERENCES}

[1] Halim Alwi, Christopher Edwards, and CheePin Tan. Fault Detection and Fault-Tolerant Control Using Sliding Modes. Springer-Verlag, 2011.

[2] F. Blanchini. Set invariance in control. Automatica, 35:1747-1767,

[3] K. Cunningham, J. V. Foster, A. M. Murch, and E. Morelli. Practical application of a subscale transport aircraft for flight research in control upset and failure conditions. In AIAA Guidance, Navigation, and Control Conference, 2008.

[4] A. Dorobantu, A. M. Murch and G. J. Balas. $h_{\infty}$ robust control design for the nasa airstar flight test vehicle. In 50th AIAA Aerospace Sciences Meeting, 2012.
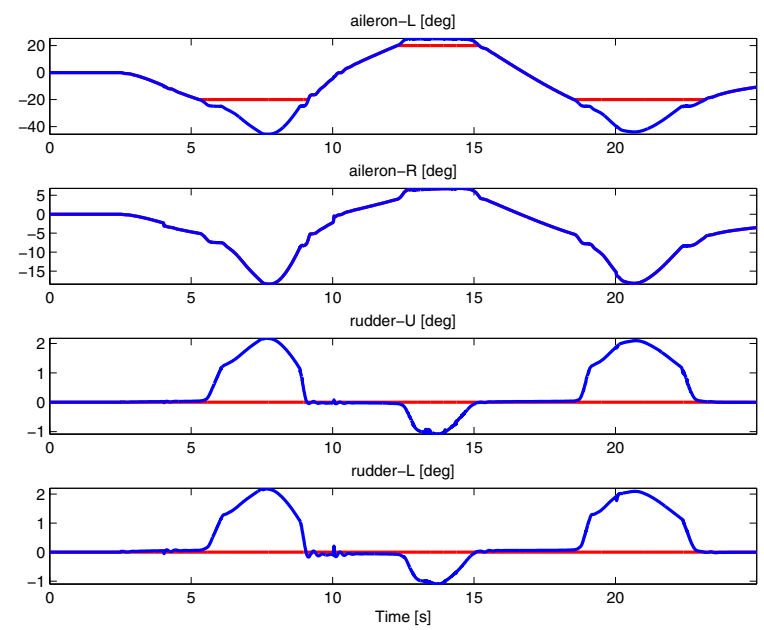

Fig. 10. First 4 control inputs in complete rudder jamming case using input allocation and fault-dependent scheduling. Blue: the modified control inputs. Red: the saturated input signals. (The rudders are switched off.)
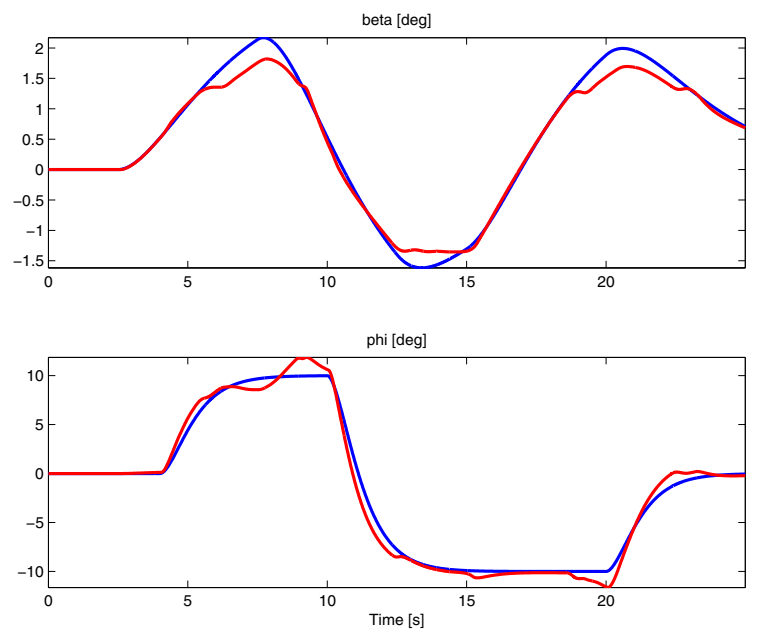

Fig. 11. Output tracking in complete rudder jamming case using input allocation and fault-dependent scheduling. Blue: reference signals filtered by $h_{\beta}, h_{\phi}$. Red: outputs of the GTM model.

[5] S. Ganguli, A. Marcos, and Gary Balas. Reconfigurable lpv control design for boeing 747-100/200 longitudinal axis. In American Control Conference, 2002. Proceedings of the 2002, volume 5, pages 36123617 vol.5, 2002.

[6] P. Goupil and A. Marcos. Advanced diagnosis for sustainable flight guidance and control: The european addsafe project. In SAE Aerospace Technology Conference and Exhibition, 2012.

[7] Philippe Goupil and Andres Marcos. Industrial benchmarking and evaluation of addsafe fdd designs. In Fault Detection, Supervision and Safety of Technical Processes (8th SAFEPROCESS), 2012.

[8] T. A. Johansen and T. I. Fossen. Control allocation - a survey. Automatica, 49(1087-1103), 2013.

[9] M. Mahmoud, J. Jiang, and Y. Zhang. Active Fault Tolerant Control System. Springer-Verlag, 2003.

[10] A. M. Murch. A flight control system architecture for the nasa airstar flight test infrastructure. In AIAA Guid ance, Navigation, and Control Conference, 2008.

[11] C. Sloth, T. Esbensen, and J. Stoustrup. Active and passive faulttolerant lpv control of wind turbines. In American Control Conference (ACC), 2010, pages 4640-4646, 2010.

[12] Marc Steinberg. A historical overview of research in reconfigurable flight control. Aerospace Control and Guidance Systems Committee Meeting No. 95, Subcomitee E:1-38, 2005.

[13] L. Zaccarian. Dynamic allocation for input redundant control systems. Automatica, 45:1431-1438, 2009.

[14] Y. Zhang and J. Jiang. Bibliographical review on reconfigurable faulttolerant control systems. Annual Reviews in Control, 32:229-252, 2008. 